\title{
Traps to the BGJT-algorithm for discrete logarithms
}

\author{
Qi Cheng, Daqing Wan and Jincheng Zhuang
}

\begin{abstract}
In the recent breakthrough paper by Barbulescu, Gaudry, Joux and Thomé, a quasi-polynomial time algorithm is proposed for the discrete logarithm problem over finite fields of small characteristic. The time complexity analysis of the algorithm is based on several heuristics presented in their paper. We show that some of the heuristics are problematic in their original forms, in particular when the field is not a Kummer extension. We propose a fix to the algorithm in non-Kummer cases, without altering the heuristic quasi-polynomial time complexity. Further study is required in order to fully understand the effectiveness of the new approach.
\end{abstract}

\section{Introduction}

Many cryptography protocols rely on hard computational number-theoretical problems for security. The discrete logarithm problem over finite fields is one of the most important candidates, besides the integer factorization problem. The hardness of discrete logarithms underpins the security of the widely adopted Diffie-Hellman key exchange protocol [5] and ElGamal's cryptosystem [6].

The state-of-the-art general-purpose methods for solving the discrete logarithm problem in finite fields are the number field sieve and the function field sieve, which originated from the index-calculus algorithm. All the algorithms run in subexponential time. Let

$$
L_{N}(\alpha)=\exp \left(O\left((\log N)^{\alpha}(\log \log N)^{1-\alpha}\right)\right) .
$$

For a finite field $\mathbf{F}_{q}$, successful efforts have been made to reduce the heuristic complexity of these algorithms from $L_{q}(1 / 2)$ to $L_{q}(1 / 3)$. See $[\mathbf{1}, \mathbf{2}, \mathbf{4}, \mathbf{9}, \mathbf{1 3}-\mathbf{1 5}, \mathbf{1 7}]$.

A sequence of breakthrough results $[8, \mathbf{1 1}, \mathbf{1 2}]$ recently on the discrete logarithm problem over finite fields culminated in the discovery of a quasi-polynomial algorithm (QPA) for small characteristic fields [3]. For a finite field $\mathbf{F}_{q^{2 k}}$ with $k<q$, the algorithm runs in heuristic time $q^{O(\log k)}$. This result, if correct, essentially removes the discrete logarithm over small characteristic fields from hard problems in cryptography. It also helps in constructing multiplicative generators in finite fields [10].

\subsection{Where does the computation really happen?}

Most serious attacks on the discrete logarithm problem over finite fields are based on smoothness of integers or polynomials. A polynomial is $m$-smooth if all its irreducible factors have degrees less than or equal to $m$. The probability that a random polynomial of degree $n$ $(\geqslant m)$ over a finite field $\mathbf{F}_{q}$ is $m$-smooth is about $(n / m)^{-n / m}[\mathbf{1 6}]$.

Received 27 February 2014; revised 23 May 2014

2010 Mathematics Subject Classification 11 Y16 (primary).

Contributed to the Algorithmic Number Theory Symposium XI, GyeongJu, Korea, 6-11 August 2014.

The research is partially supported by the 973 Program (grant no. 2013CB834201) and NSF (grant no. CCF-0830522) for QC and JZ. The second author was partially supported by a Simons Fellowship. 
Suppose that we need to compute discrete logarithms in the field $\mathbf{F}_{q^{2 k}}$ where $q>k>1$. An important technique in [3], based on smooth polynomials, is to find a nice ring generator $\zeta$ of $\mathbf{F}_{q^{2 k}}=\mathbf{F}_{q^{2}}[\zeta]$ over $\mathbf{F}_{q^{2}}$ satisfying

$$
x^{q}=h_{0}(x) / h_{1}(x),
$$

where $h_{1}$ and $h_{0}$ are polynomials of very small degree. In many parts of the computation, polynomial degrees can be dropped quickly by replacing $x^{q}$ with $h_{0}(x) / h_{1}(x)$, which allows an effective attack based on smoothness.

The main issue with this approach is that the computation really takes place in the ring $\mathbf{F}_{q^{2}}[x] /\left(x^{q} h_{1}(x)-h_{0}(x)\right)$, while in the analysis of [3], the computation is assumed to be in $\mathbf{F}_{q^{2}}[x] /(f(x))$, where $f(x)$ is the minimal polynomial of $\zeta$ over $\mathbf{F}_{q^{2}}$. Since $f(x)$ divides $x^{q} h_{1}(x)-$ $h_{0}(x)$, there is a natural surjective ring homomorphism

$$
\mathbf{F}_{q^{2}}[x] /\left(x^{q} h_{1}(x)-h_{0}(x)\right) \rightarrow \mathbf{F}_{q^{2}}[x] /(f(x)) .
$$

But the former ring, which is a direct sum of the latter field (if $f(x)$ is a simple factor of $\left.x^{q} h_{1}(x)-h_{0}(x)\right)$ and a few other rings, is much larger in many cases. The computation thus can be affected by the other rings, rendering several conjectures in $[3,12]$ problematic.

\subsection{Our work}

Interestingly, for the Kummer extension of the form $\mathbf{F}_{q^{2}}[x] /\left(x^{q-1}-a\right)$, everything is fine. This is because the difference between the ring $\mathbf{F}_{q^{2}}[x] /\left(x^{q}-a x\right)$ and the field is rather small. The discrete logarithm of $x$, which is a zero divisor in the former ring, can be computed easily in the latter field, since it belongs to a subgroup of small order (dividing $\left.(q-1)\left(q^{2}-1\right)\right)$ in the field. This is consistent with all announced practical implementations.

However, in the case of more difficult non-Kummer extensions, we discover that there are multiple problems. First, if $x^{q} h_{1}(x)-h_{0}(x)$ has linear factors over $\mathbf{F}_{q^{2}}$, the discrete logarithms of these linear factors cannot be computed in polynomial time, invalidating a basic assumption in [3]. One can verify that most of polynomials given in [12, Table 1] have linear factors. Second, even at the stage of finding discrete logarithms of linear elements, we show that there are additional serious restrictions on the choice of $h_{0}$ and $h_{1}$. For example, if $x^{q} h_{1}(x)-h_{0}(x)$ has another irreducible factor over $\mathbf{F}_{q^{2}}$ of degree $k_{i}$ satisfying $\operatorname{gcd}\left(k_{i}, k\right)>1$, we do not see how the algorithm can work. See Theorem 2 for details. We propose to select $h_{0}$ and $h_{1}$ such that $x^{q} h_{1}(x)-h_{0}(x)$ has only one irreducible factor $f(x)$ over $\mathbf{F}_{q^{2}}$ of degree $k$, and all other irreducible factors over $\mathbf{F}_{q^{2}}$ have degrees bigger than 1 and relatively prime to $k$. Under these assumptions, we give an algorithm which will find the discrete logarithm of any linear element in polynomial time, under a heuristic assumption supported by our theoretical results and numerical data.

For a non-linear element, a clever idea, the so-called QPA-descent, was proposed in [3] to reduce its degree, until its relation to linear factors can be found. While the above two problems about linear factors can be fixed under our newly improved heuristic assumptions, another serious problem is that there are traps in the QPA-descent. For these traps, the QPAdescent described in [3] will not work at all. They will also block the descent of other elements, hence severely affecting the usefulness of the new algorithm. We propose a descent strategy that avoids the traps, without altering the quasi-polynomial time complexity. The modified algorithm is also heuristic. We have done a few numerical studies to confirm the heuristic.

In summary, for large non-Kummer fields, we believe that the basic idea behind the new approach should still work, but the problem can be significantly more subtle than previously thought and further study needs to be conducted in order to fully understand the effect of the new algorithm. 


\section{Finding the discrete logarithm of the linear factors}

We first review the new algorithm in [3]. Suppose that the discrete logarithm is sought over the field $\mathbf{F}_{q^{2 k}}$ with $k<q$. For other small characteristic fields, such as $\mathbf{F}_{p^{k}}(p<k)$, one first embeds it into a slightly larger field,

$$
\mathbf{F}_{p^{k}} \rightarrow \mathbf{F}_{q^{k}} \rightarrow \mathbf{F}_{q^{2 k}}
$$

where $q=p^{\left\lceil\log _{p} k\right\rceil}$. A quasi-polynomial time algorithm for $\mathbf{F}_{q^{2 k}}$ implies a quasi-polynomial time algorithm for $\mathbf{F}_{p^{k}}$. We assume that

$$
\mathbf{F}_{q^{2 k}}=\mathbf{F}_{q^{2}}[\zeta]
$$

where $\zeta^{q}=h_{0}(\zeta) / h_{1}(\zeta)$. Here $h_{0}$ and $h_{1}$ are polynomials over $\mathbf{F}_{q^{2}}$ relatively prime to each other, and of a constant degree. In particular, $\operatorname{deg}\left(h_{0}\right)<q+\operatorname{deg}\left(h_{1}\right)$. To find such a nice ring generator $\zeta$, one searches over all the polynomials $h_{0}(x)$ and $h_{1}(x)$ of a constant degree in $\mathbf{F}_{q^{2}}[x]$, until $h_{1}(x) x^{q}-h_{0}(x)$ has an irreducible factor $f(x)$ of degree $k$ with multiplicity 1 . Let the factorization be

$$
x^{q} h_{1}(x)-h_{0}(x)=f(x) \prod_{i=1}^{l}\left(f_{i}(x)\right)^{a_{i}}
$$

where the polynomials $f(x)$ and $f_{i}(x)$ are irreducible and pairwise prime. Denote the degree of $f_{i}(x)$ by $k_{i}$.

REMARK 1. In practice, it is enough to search only a quadratic polynomial $h_{0}$ (not necessarily monic) and a monic linear polynomial $h_{1}$ in $\mathbf{F}_{q^{2}}[x]$. However, proving the existence of such polynomials for any constant degree such that $x^{q} h_{1}(x)-h_{0}(x)$ has the desired factorization pattern seems to be out of reach by current techniques.

For simplicity we assume that $h_{1}(x)$ is monic and linear. Most of the known algorithms start by computing the discrete logarithms of elements in a special set called a factor base, which usually contains small integers, or low-degree polynomials. In the new approach $[\mathbf{3}, \mathbf{1 2}]$, the factor base consists of the linear polynomials $\zeta+\alpha$ for all $\alpha \in \mathbf{F}_{q^{2}}$, and an algorithm is designed to compute the discrete logarithms of all the elements in the factor base. It is conjectured that this algorithm runs in polynomial time. One starts the algorithm with the identity,

$$
\prod_{\alpha \in \mathbf{F}_{q}}(x-\alpha)=x^{q}-x
$$

One then applies the Möbius transformation

$$
x \mapsto \frac{a x+b}{c x+d},
$$

where the matrix $m=\left(\begin{array}{ll}a & b \\ c & d\end{array}\right) \in \mathbf{F}_{q^{2}}^{2 \times 2}$ is non-singular. We have

$$
\prod_{\alpha \in \mathbf{F}_{q}}\left(\frac{a x+b}{c x+d}-\alpha\right)=\left(\frac{a x+b}{c x+d}\right)^{q}-\frac{a x+b}{c x+d} .
$$

Clearing the denominator,

$$
\begin{aligned}
(c x+d) & \prod_{\alpha \in \mathbf{F}_{q}}((a x+b)-\alpha(c x+d)) \\
& =(a x+b)^{q}(c x+d)-(a x+b)(c x+d)^{q} \\
& =\left(a^{q} x^{q}+b^{q}\right)(c x+d)-(a x+b)\left(c^{q} x^{q}+d^{q}\right) .
\end{aligned}
$$


Multiplying both sides by $h_{1}(x)$ and replacing $x^{q} h_{1}(x)$ by $h_{0}(x)$, we obtain

$$
\begin{aligned}
h_{1}(x) & (c x+d) \prod_{\alpha \in \mathbf{F}_{q}}((a x+b)-\alpha(c x+d)) \\
= & \left(a^{q} h_{0}(x)+b^{q} h_{1}(x)\right)(c x+d)-(a x+b)\left(c^{q} h_{0}(x)+d^{q} h_{1}(x)\right) \\
& \left(\bmod x^{q} h_{1}(x)-h_{0}(x)\right) .
\end{aligned}
$$

If the right-hand side can be factored into a product of linear factors over $\mathbf{F}_{q^{2}}$, we obtain a relation of the form

$$
\lambda^{e_{0}} \prod_{i=1}^{q^{2}}\left(x+\alpha_{i}\right)^{e_{i}}=\prod_{i=1}^{q^{2}}\left(x+\alpha_{i}\right)^{e_{i}^{\prime}} \quad\left(\bmod x^{q} h_{1}(x)-h_{0}(x)\right),
$$

where $\lambda$ is a multiplicative generator of $\mathbf{F}_{q^{2}}, \alpha_{1}=0, \alpha_{2}, \alpha_{3}, \ldots, \alpha_{q^{2}}$ is a natural ordering of elements in $\mathbf{F}_{q^{2}}$, and the $e_{i}$ and $e_{i}^{\prime}$ are non-negative integers.

Using the same notation as in [3], let $\mathcal{P}_{q}$ be a set of representatives of the left cosets of $\mathrm{PGL}_{2}\left(\mathbf{F}_{q}\right)$ in $\mathrm{PGL}_{2}\left(\mathbf{F}_{q^{2}}\right)$. Note that the cardinality of $\mathcal{P}_{q}$ is $q^{3}+q$. It was shown in $[\mathbf{3}]$ that the matrices in the same coset produce the same relation (2.2).

Suppose that, for some $1 \leqslant g \leqslant q^{2}, \zeta+\alpha_{g}$ is a known multiplicative generator of $\mathbf{F}_{q^{2}}[\zeta]=$ $\mathbf{F}_{q^{2}}[x] /(f(x))$. Since (2.2) also holds modulo $f(x)$, taking the discrete logarithm with respect to the base $\zeta+\alpha_{g}$, we obtain

$$
e_{0} \log _{\zeta+\alpha_{g}} \lambda+\sum_{1 \leqslant i \leqslant q^{2}, i \neq g}\left(e_{i}-e_{i}^{\prime}\right) \log _{\zeta+\alpha_{g}}\left(\zeta+\alpha_{i}\right) \equiv e_{g}^{\prime}-e_{g} \quad\left(\bmod q^{2 k}-1\right)
$$

The above equation gives us a linear relation among the discrete logarithms of linear factors. One hopes to collect enough relations such that the linear system formed by those relations is non-singular over $\mathbf{Z} /\left(q^{2 k}-1\right) \mathbf{Z}$. It allows us to solve $\log _{\zeta+\alpha_{g}}\left(\zeta+\alpha_{i}\right)$ for all the $\zeta+\alpha_{i}$ in the factor base.

However, if, for some $1 \leqslant z \leqslant q^{2}$,

$$
\left(x+\alpha_{z}\right) \mid x^{q} h_{1}(x)-h_{0}(x),
$$

the algorithm will not likely compute $\log _{\zeta+\alpha_{g}}\left(\zeta+\alpha_{z}\right)$. This is because $x+\alpha_{z}$ is zero or nilpotent (without loss of generality, let $\left.f_{1}=x+\alpha_{z}\right)$ in the $\mathbf{F}_{q^{2}}[x] /\left(\left(x+\alpha_{z}\right)^{a_{1}}\right)$ component of the ring

$$
\mathbf{F}_{q^{2}}[x] /\left(x^{q} h_{1}(x)-h_{0}(x)\right)=\mathbf{F}_{q^{2}}[x] /(f(x)) \oplus \bigoplus_{i=1}^{l} \mathbf{F}_{q^{2}}[x] /\left(f_{i}(x)^{a_{i}}\right) .
$$

Hence in (2.2), if $e_{z}>0, e_{z}^{\prime}$ is positive as well. Most likely we will have $e_{z}=e_{z}^{\prime}$, so the coefficient for $\log _{\zeta+\alpha_{g}}\left(\zeta+\alpha_{z}\right)$ in (2.3) will always be 0 .

REMARK 2. If $e_{z}^{\prime}>e_{z} \geqslant 1$, it is possible to compute $\log _{\zeta+\alpha_{g}}\left(\zeta+\alpha_{z}\right)$. However, this requires the low-degree polynomial in the right-hand side of (2.2) to have the factor $\left(x+\alpha_{z}\right)^{2}$, which is unlikely. Our numerical data confirm that it never happens when $q$ is sufficiently large.

To compute the discrete logarithm of $\zeta+\alpha_{z}$, we have to use additional relations which hold for the field $\mathbf{F}_{q^{2}}[\zeta]$ but may not hold for the bigger ring $\mathbf{F}_{q^{2}}[x] /\left(x^{q} h_{1}(x)-h_{0}(x)\right)$. 
The equation

$$
\left(\zeta+\alpha_{z}\right)^{q^{2 k}-1}=1
$$

is such an example. But this does not help in computing its discrete logarithm in the field $\mathbf{F}_{q^{2}}[\zeta]$, if it is the only relation involving $\zeta+\alpha_{z}$.

REMARK 3. An exception is in the case of a Kummer extension, where the zero divisor $x$ in the ring has a small order in the field.

In general, it is hard to find useful additional relations for $x+\alpha_{z}$, since for the algorithm to work, it is essential that we replace $x^{q}$ by $h_{0}(x) / h_{1}(x)$ (not replace $f(x)$ by zero) in the relation generating stage. Hence it is not clear that the discrete logarithm of $\zeta+\alpha_{z}$ can be computed in polynomial time, invalidating a conjecture in [3].

\section{The tale of two lattices}

To fix the above problem in a non-Kummer case, we can either change our factor base to not include the linear factors of $x^{q} h_{1}(x)-h_{0}(x)$, or we can search for $h_{0}$ and $h_{1}$ such that $x^{q} h_{1}(x)-h_{0}(x)$ does not have linear factors. In the following discussion, we will assume that $x^{q} h_{1}(x)-h_{0}(x)$ has no linear factor for simplicity. That is,

$$
k_{i}:=\operatorname{deg}\left(f_{i}\right) \geqslant 2 \quad(1 \leqslant i \leqslant l) .
$$

In this case, the linear factors $x+\alpha_{i}$ are invertible in the ring $\mathbf{F}_{q^{2}}[x] /\left(x^{q} h_{1}(x)-h_{0}(x)\right)$ and equation (2.2) reduces to

$$
\lambda^{e_{0}} \prod_{i=1}^{q^{2}}\left(x+\alpha_{i}\right)^{e_{i}-e_{i}^{\prime}}=1\left(\bmod x^{q} h_{1}(x)-h_{0}(x)\right) .
$$

We define two fundamental lattices in $\mathbf{Z}^{q^{2}+1}$ :

$$
\begin{aligned}
& \mathcal{L}_{1}=\left\{\left(e_{0}, e_{1}, \ldots, e_{q^{2}}\right) \mid \lambda^{e_{0}} \prod_{i=1}^{q^{2}}\left(x+\alpha_{i}\right)^{e_{i}}=1(\bmod f(x))\right\}, \\
& \mathcal{L}_{2}=\left\{\left(e_{0}, e_{1}, \ldots, e_{q^{2}}\right) \mid \lambda^{e_{0}} \prod_{i=1}^{q^{2}}\left(x+\alpha_{1}\right)^{e_{i}}=1\left(\bmod x^{q} h_{1}(x)-h_{0}(x)\right)\right\} .
\end{aligned}
$$

It is easy to see that $\mathcal{L}_{2} \subseteq \mathcal{L}_{1}$. Consider the group homomorphism

$$
\psi_{1}: \mathbf{Z}^{q^{2}+1} \rightarrow\left(\mathbf{F}_{q^{2}}[x] /(f(x))\right)^{*}
$$

given by

$$
\left(e_{0}, e_{1}, \ldots, e_{q^{2}}\right) \mapsto \lambda^{e_{0}} \prod_{i=1}^{q^{2}}\left(x+\alpha_{i}\right)^{e_{i}} .
$$

The group homomorphism $\psi_{2}$ is defined in the same way, except that modulo $f(x)$ is replaced by modulo $\left(x^{q} h_{1}(x)-h_{0}(x)\right)$, respectively.

Theorem 1. If $\operatorname{deg}\left(h_{1}\right) \leqslant 2$, then the maps $\psi_{1}$ and $\psi_{2}$ are surjective. 
Proof. It is enough to prove that $\psi_{2}$ is surjective. If not, the image $H$ of $\psi_{2}$ would be a proper subgroup of $\left(\mathbf{F}_{q^{2}}[x] /\left(x^{q} h_{1}(x)-h_{0}(x)\right)\right)^{*}$. We can then choose a non-trivial character $\chi$ of $\left(\mathbf{F}_{q^{2}}[x] /\left(x^{q} h_{1}(x)-h_{0}(x)\right)\right)^{*}$ which is trivial on the subgroup $H$. Since $\chi$ is trivial on $H$ which contains $\mathbf{F}_{q^{2}}^{*}$, we can use the Weil bound as given in $[\mathbf{1 8}$, Theorem 2.1] and deduce that

$$
1+q^{2}=\left|1+\sum_{\alpha \in \mathbf{F}_{q^{2}}} \chi(x+\alpha)\right| \leqslant\left(q+\operatorname{deg}\left(h_{1}\right)-2\right) \sqrt{q^{2}} \leqslant q^{2} .
$$

This is a contradiction. It follows that $\psi_{2}$ must be surjective.

Note that the determination of the factor base discrete logarithms corresponds to the computation of the Smith normal form of $\mathcal{L}_{1}$, which has been observed in many places, for example [7]. In this application it is important that $\psi_{1}$ is surjective. As a corollary, we deduce the following result.

Corollary 1. If $\operatorname{deg}\left(h_{1}\right) \leqslant 2$, then:

- the group $\mathbf{Z}^{q^{2}+1} / \mathcal{L}_{1}$ is isomorphic to the cyclic group $\mathbf{Z} /\left(q^{2 k}-1\right) \mathbf{Z}$;

- the group $\mathbf{Z}^{q^{2}+1} / \mathcal{L}_{2}$ is isomorphic to

$$
\left.\mathbf{Z} /\left(q^{2 k}-1\right) \mathbf{Z} \oplus \bigoplus_{i=1}^{l} \mathbf{Z} /\left(q^{2 k_{i}}-1\right) \mathbf{Z} \bigoplus \text { (a finite } p \text {-group }\right)
$$

In particular, the group $\mathbf{Z}^{q^{2}+1} / \mathcal{L}_{2}$ is not cyclic when $l \geqslant 1$. The relation generation stage only gives lattice vectors in $\mathcal{L}_{2}$, which is far from $\mathcal{L}_{1}$ if $l \geqslant 1$. Thus, we need to add more relations to $\mathcal{L}_{2}$ in order to get close to $\mathcal{L}_{1}$.

Since $\lambda^{q^{2}-1}=1$, the vector $\left(q^{2}-1,0, \ldots, 0\right)$ is automatically in $\mathcal{L}_{2}$. Let $\mathcal{L}_{2}^{*}$ be the lattice in $\mathbf{Z}^{q^{2}+1}$ generated by $\mathcal{L}_{2}$ and the $q^{2}$ vectors

$$
\left(0, q^{2 k}-1,0, \ldots, 0\right), \ldots,\left(0,0, \ldots, 0, q^{2 k}-1\right),
$$

corresponding to the relations $\left(x+\alpha_{i}\right)^{q^{2 k}-1}=1$ modulo $f(x)$ for $\alpha_{i} \in \mathbf{F}_{q^{2}}$. It is clear that

$$
\mathcal{L}_{2}^{*}=\mathcal{L}_{2}+\left(q^{2 k}-1\right) \mathbf{Z}^{q^{2}+1} .
$$

The next result gives the group structure for the quotient $\mathbf{Z}^{q^{2}+1} / \mathcal{L}_{2}^{*}$.

TheOrem 2. For $\operatorname{deg}\left(h_{1}\right) \leqslant 2$, there is a group isomorphism

$$
\mathbf{Z}^{q^{2}+1} / \mathcal{L}_{2}^{*} \cong \mathbf{Z} /\left(q^{2 k}-1\right) \mathbf{Z} \oplus \bigoplus_{1 \leqslant i \leqslant l} \mathbf{Z} /\left(q^{2 \operatorname{gcd}\left(k, k_{i}\right)}-1\right) \mathbf{Z}
$$

Proof. Recall that

$$
\mathbf{Z}^{q^{2}+1} / \mathcal{L}_{2} \cong A \stackrel{\text { def }}{=} \mathbf{Z} /\left(q^{2 k}-1\right) \mathbf{Z} \oplus \bigoplus_{i=1}^{l} \mathbf{Z} /\left(q^{2 k_{i}}-1\right) \mathbf{Z} \bigoplus \text { (a finite } p \text {-group). }
$$

It is clear that

$$
A /\left(q^{2 k}-1\right) A \cong \mathbf{Z} /\left(q^{2 k}-1\right) \mathbf{Z} \oplus \bigoplus_{1 \leqslant i \leqslant l} \mathbf{Z} /\left(q^{2 \operatorname{gcd}\left(k_{i}, k\right)}-1\right) \mathbf{Z}
$$


The kernel of the surjective composed homomorphism

$$
\mathbf{Z}^{q^{2}+1} \longrightarrow \mathbf{Z}^{q^{2}+1} / \mathcal{L}_{2} \cong A \longrightarrow A /\left(q^{2 k}-1\right) A
$$

is precisely $\mathcal{L}_{2}+\left(q^{2 k}-1\right) \mathbf{Z}^{q^{2}+1}=\mathcal{L}_{2}^{*}$. The desired isomorphism follows.

If $\operatorname{gcd}\left(k_{i}, k\right)>1$ for some $i$, then $\mathcal{L}_{2}^{*}$ is still far from $\mathcal{L}_{1}$. We would like $\mathcal{L}_{2}^{*}$ to be as close to $\mathcal{L}_{1}$ as possible in a smooth sense. For us, the more interesting case is the following corollary.

Corollary 2. Let $\operatorname{deg}\left(h_{1}\right) \leqslant 2$. If $\operatorname{gcd}\left(k_{i}, k\right)=1$ for all $1 \leqslant i \leqslant l$, we have an isomorphism

$$
\mathbf{Z}^{q^{2}+1} / \mathcal{L}_{2}^{*} \cong \mathbf{Z} /\left(q^{2 k}-1\right) \mathbf{Z} \oplus\left(\mathbf{Z} /\left(q^{2}-1\right) \mathbf{Z}\right)^{l} .
$$

This corollary shows that under the same assumption, the lattice $\mathcal{L}_{2}^{*}$ is a smooth approximation of $\mathcal{L}_{1}$ in the sense that the quotient $\mathcal{L}_{1} / \mathcal{L}_{2}^{*}$ is a direct sum of small-order cyclic groups.

The algorithm to compute the discrete logarithms in the factor base essentially samples vectors from the lattice $\mathcal{L}_{2}$. Let $\mathbf{r}_{1}, \mathbf{r}_{2}, \ldots$, be the vectors in $\mathcal{L}_{2}$ obtained by the relation finding algorithm, that is, from the relations in (3.1). Let $\hat{\mathcal{L}_{2}}$ be the lattice generated by those vectors. Let $\hat{\mathcal{L}_{1}}$ be the lattice generated by $\hat{\mathcal{L}_{2}}$ and the following $q^{2}+1$ vectors:

$$
\left(q^{2}-1,0, \ldots, 0\right),\left(0, q^{2 k}-1,0, \ldots, 0\right), \ldots,\left(0,0, \ldots, 0, q^{2 k}-1\right) .
$$

Computing the Hermite (or Smith) normal form of $\hat{\mathcal{L}}_{1}$ is equivalent to solving the linear system $\hat{\mathcal{L}}_{2}$ in the ring $\mathbf{Z} /\left(q^{2 k}-1\right) \mathbf{Z}$. It is in general difficult to find bases for the two lattices $\mathcal{L}_{1}$ and $\mathcal{L}_{2}$ directly. One can think of $\hat{\mathcal{L}_{1}}$ and $\hat{\mathcal{L}_{2}}$ as approximations of $\mathcal{L}_{1}$ and $\mathcal{L}_{2}$, respectively. These approximations can be computed by the polynomial time algorithm. Obviously,

$$
\begin{aligned}
\hat{\mathcal{L}_{2}} & \subseteq \mathcal{L}_{2} \subseteq \\
& \subseteq \hat{\mathcal{L}}_{1} \subseteq
\end{aligned}
$$

These inclusions induce surjective group homomorphisms

$$
\begin{aligned}
\mathbf{Z}^{q^{2}+1} / \hat{\mathcal{L}_{2}} & \rightarrow \mathbf{Z}^{q^{2}+1} / \mathcal{L}_{2} \rightarrow \mathbf{Z}^{q^{2}+1} / \mathcal{L}_{2}^{*} \rightarrow \mathbf{Z}^{q^{2}+1} / \mathcal{L}_{1} . \\
& \rightarrow \mathbf{Z}^{q^{2}+1} / \hat{\mathcal{L}_{1}} \rightarrow
\end{aligned}
$$

If $\mathbf{Z}^{q^{2}+1} / \hat{\mathcal{L}_{2}}$ is cyclic, then its quotient $\mathbf{Z}^{q^{2}+1} / \mathcal{L}_{2}$ will be cyclic. This is false if $l \geqslant 1$, as we have seen before. Similarly, $\mathbf{Z}^{q^{2}+1} / \hat{\mathcal{L}_{1}}$ is not cyclic as its quotient $\mathbf{Z}^{q^{2}+1} / \mathcal{L}_{2}^{*}$ is not cyclic if $l \geqslant 1$. It seems reasonable to hope that $\hat{\mathcal{L}}_{1}$ is a good approximation to $\mathcal{L}_{2}^{*}$ in the sense that the quotient $\mathcal{L}_{2}^{*} / \hat{\mathcal{L}}_{1}$ is a direct sum of small-order cyclic groups. In the interesting case where $\operatorname{gcd}\left(k, k_{i}\right)=1$ for all $1 \leqslant i \leqslant l$, our numerical data suggest the following highly plausible heuristic.

HeURistic 1. Assume that $x^{q} h_{1}(x)-h_{0}(x)$ does not have linear factors, and $\operatorname{gcd}\left(k, k_{i}\right)=1$ for all $1 \leqslant i \leqslant l$. Then in the Smith normal form of $\hat{\mathcal{L}}_{1}$, the diagonal elements are

$$
1,1, \ldots, 1, s_{1}, \ldots, s_{t}, q^{2 k}-1,
$$

where, for $1 \leqslant i \leqslant t, s_{i}>1$ and $s_{i} \mid q^{2}-1$.

Assuming the heuristic, $\mathbf{Z}^{q^{2}+1} / \hat{\mathcal{L}_{1}}$ is not much bigger than $\mathbf{Z}^{q^{2}+1} / \mathcal{L}_{1}$, namely,

$$
\mathbf{Z}^{q^{2}+1} / \hat{\mathcal{L}_{1}} \cong \mathbf{Z} / s_{1} \mathbf{Z} \oplus \mathbf{Z} / s_{2} \mathbf{Z} \oplus \ldots \oplus \mathbf{Z} / s_{t} \mathbf{Z} \oplus \mathbf{Z} /\left(q^{2 k}-1\right) \mathbf{Z} .
$$


We can find a generator for each component, as a product of linear polynomials from the computation of the Smith normal form. Suppose that, for $1 \leqslant i \leqslant t$, the generator for the $i$ th component is

$$
\lambda^{e_{i 0}} \prod_{1 \leqslant j \leqslant q^{2}}\left(x+\alpha_{j}\right)^{e_{i j}}
$$

Since $s_{i} \mid q^{2}-1$, the above generator belongs to $\mathbf{F}_{q^{2}}$ in $\mathbf{F}_{q^{2}}[x] /(f(x))$. Assuming that it is $\lambda^{e^{\prime}}$, we have

$$
\lambda^{e_{i 0}-e_{i 0}^{\prime}} \prod_{1 \leqslant j \leqslant q^{2}}\left(x+\alpha_{j}\right)^{e_{i j}}=1(\bmod f(x)) .
$$

There are $t$ such relations. Adding them to $\hat{\mathcal{L}_{1}}$, we will finally arrive at the lattice $\mathcal{L}_{1}$. This allows us to find a generator for $\left(\mathbf{F}_{q^{2}}[x] /(f(x))\right)^{*}$, and to solve the discrete logarithms for the factor base, with respect to this generator.

\section{The trap to the QPA-descent}

Now we review the QPA-descent. Suppose that we need to compute the discrete logarithm of $W(\zeta) \in \mathbf{F}_{q^{2 k}}[\zeta]$, where $W$ is a polynomial over $\mathbf{F}_{q^{2}}$ of degree $w>1$. The QPA-descent, first proposed in [3], is to represent $W(\zeta)$ as a product of elements of smaller degree, for example up to $w / 2$, in the field $\mathbf{F}_{q^{2}}[x] /(f(x))$. To do this, one again starts with the identity:

$$
\prod_{\alpha \in \mathbf{F}_{q}}(x-\alpha)=x^{q}-x .
$$

One then applies the transformation

$$
x \mapsto \frac{a W(x)+b}{c W(x)+d},
$$

where the matrix $m=\left(\begin{array}{ll}a & b \\ c & d\end{array}\right) \in \mathbf{F}_{q^{2}}^{2 \times 2}$ is non-singular. We have

$$
\prod_{\alpha \in \mathbf{F}_{q}}\left(\frac{a W(x)+b}{c W(x)+d}-\alpha\right)=\left(\frac{a W(x)+b}{c W(x)+d}\right)^{q}-\frac{a W(x)+b}{c W(x)+d} .
$$

Clearing the denominator,

$$
\begin{aligned}
& (c W(x)+d) \prod_{\alpha \in \mathbf{F}_{q}}((a W(x)+b)-\alpha(c W(x)+d)) \\
& \quad=(a W(x)+b)^{q}(c W(x)+d)-(a W(x)+b)(c W(x)+d)^{q} \\
& \quad=\left(a^{q} \tilde{W}\left(x^{q}\right)+b^{q}\right)(c W(x)+d)-(a W(x)+b)\left(c^{q} \tilde{W}\left(x^{q}\right)+d^{q}\right),
\end{aligned}
$$

where $\tilde{W}(x)$ is a polynomial obtained by raising the coefficients of $W(x)$ to the $q$ th power. Replacing $x^{q}$ with $h_{0}(x) / h_{1}(x)$, we obtain

$$
\begin{aligned}
(c W(x)+d) & \prod_{\alpha \in \mathbf{F}_{q}}((a W(x)+b)-\alpha(c W(x)+d)) \\
= & \left(a^{q} \tilde{W}\left(h_{0}(x) / h_{1}(x)\right)+b^{q}\right)(c W(x)+d) \\
- & (a W(x)+b)\left(c^{q} \tilde{W}\left(h_{0}(x) / h_{1}(x)\right)+d^{q} h_{1}(x)\right) \\
& \left(\bmod x^{q} h_{1}(x)-h_{0}(x)\right) .
\end{aligned}
$$


It was shown in $[\mathbf{3}]$ that matrices in the same left coset of $\mathrm{PGL}_{2}\left(\mathbf{F}_{q}\right)$ of $\mathrm{PGL}_{2}\left(\mathbf{F}_{q^{2}}\right)$ generate the same equations. The denominator of the right-hand side is a power of $h_{1}(x)$. Denote the numerator of the right-hand-side polynomial by $N_{m, W}(x)$. If the polynomial $N_{m, W}(x)$ is $w / 2$ smooth, namely, it can be factored completely into a product of irreducible factors over $\mathbf{F}_{q^{2}}$, all having degree $w / 2$ or less, we obtain a relation of the form

$$
\prod_{i=1}^{q^{2}}\left(W(x)+\alpha_{i}\right)^{e_{i}}=\lambda^{e_{0}} \prod_{g(x) \in S} g(x)^{e_{g}^{\prime}}\left(\bmod x^{q} h_{1}(x)-h_{0}(x)\right)
$$

where $S \subseteq \mathbf{F}_{q^{2}}[x]$ is a set of monic polynomials of degrees less than $w / 2$ and with cardinality at most $3 w$. Denote the vector $\left(e_{1}, e_{2}, \ldots, e_{q^{2}}\right)$ by $\mathbf{v}_{m}$. Note that it is a binary vector, and it is independent of $W(x)$. Collecting a sufficient number of relations will allow us to represent $W(x)$ as a product of elements of smaller degrees. This process is the QPA-descent. A heuristic, from [3], is that by repeating the process, one can represent any element in $\mathbf{F}_{q^{2}}[x] /(f(x))$ as a product of linear factors. Combining it with the fact that the discrete logarithms of the linear factors are known, one solves the discrete logarithm for any element.

However, the descent will not work if $W(x)$ is a factor of $x^{q} h_{1}(x)-h_{0}(x)$. Recall that $\alpha_{1}=0$.

TheOREM 3. If $W(x) \mid x^{q} h_{1}(x)-h_{0}(x), e_{1}$ will always be 0 in (4.1).

In other words, if $W(x)$ is a factor of $x^{q} h_{1}(x)-h_{0}(x)$, then it will never appear in the left-hand side of (4.1) as a factor. So the descent for $W(\zeta)$ is not possible.

Proof. The polynomial $W(x)$ is a zero divisor in the ring $\mathbf{F}_{q^{2}}[x] /\left(x^{q} h_{1}(x)-h_{0}(x)\right)$. Hence if $W(x)$ appears in the left-hand side of (4.1) as a factor, it will also appear in the right-hand side. This contradicts the requirement that the factors in the right-hand side have degrees smaller than the degree of $W(x)$.

Note that the trap factor $W(\zeta)$ can appear in the descent paths of other elements, which essentially blocks the descents. This is especially troublesome if $x^{q} h_{1}(x)-h_{0}(x)$ has many small-degree factors.

\section{The trap-avoiding descent}

Having discovered traps for the original QPA-descent, how can we work around them? From the above discussion, we assume that we work in a non-Kummer extension, and the polynomial $x^{q} h_{1}(x)-h_{0}(x)$ with the factorization as (2.1) satisfies:

- $\operatorname{deg}\left(h_{0}\right) \leqslant 2, \operatorname{deg}\left(h_{1}\right) \leqslant 1$;

- $k_{i}>1$ for all $1 \leqslant i \leqslant l$ (in other words, it is free of linear factors);

- $\operatorname{gcd}\left(k, k_{i}\right)=1$ for all $1 \leqslant i \leqslant l$.

In the most interesting case, where $k$ is a prime, our numerical data show that the above requirements can be easily satisfied.

HeURISTiC 2. Let $q$ be a prime power and $k<q$ be a prime. Then there exist polynomials $h_{0}$ and $h_{1}$ satisfying the above requirements.

Assume that the discrete logarithms of all linear polynomials have been computed. Suppose that we need to compute the discrete logarithm of $W(\zeta)$, where $W(x)$ is an irreducible polynomial of degree less than $k$, and it is relatively prime to $f(x)$. If $W(x) \mid x^{q} h_{1}(x)-h_{0}(x)$, we will search for an integer $i$ such that $W(x)^{i}(\bmod f(x))$ is relatively prime to $x^{q} h_{1}(x)-h_{0}(x)$. Such $i$ can be found easily by a random process. 
Now we can assume that $\operatorname{gcd}\left(W(x), x^{q} h_{1}(x)-h_{0}(x)\right)=1$. If there are not many traps, we will use a trap-avoiding strategy for the descent. The basic idea is simple. Whenever we find a relation (4.1), we will not use it unless the right-hand side is relatively prime to $x^{q} h_{1}(x)-h_{0}(x)$.

Definition 1. Define the trap-avoiding descent lattice $\mathcal{L}(W)$ associated with $W(x)$ to be generated by

$$
\left\{\mathbf{v}_{m} \mid N_{m, W} \text { is } w / 2 \text {-smooth, and } \operatorname{gcd}\left(N_{m, W}, x^{q} h_{1}(x)-h_{0}(x)\right)=1\right\} .
$$

Note that we use fewer relations than $[\mathbf{3}]$ does, since we have to avoid traps. If the vector $(1,0, \ldots, 0)$ is in the trap-avoiding descent lattice of $W(x)$, then $W(x)$ can be written as a product of low-degree polynomials in $\mathbf{F}_{q^{2}}[x] /(f(x))$ that are not traps. We believe that the following heuristic is very likely to be true.

Heuristic 3. The trap-avoiding descent lattice for $W(x)$ contains the vector $(1,0, \ldots, 0)$ if $\operatorname{gcd}\left(W(x), x^{q} h_{1}(x)-h_{0}(x)\right)=1$.

To provide theoretical evidence, we will show that $(1,0, \ldots, 0)$ is in its superlattice that is generated by $\mathbf{v}_{m}$ for all $m \in \mathcal{P}_{q}$, regardless of whether $N_{m, W}(x)$ is $w / 2$-smooth or not. This is a slight improvement over $[\mathbf{3}]$, where it is proved that $\left(q^{3}-q, 0, \ldots, 0\right)$ is in the superlattice. To proceed, we first give some definitions following [3]. There are two matrices in consideration. The matrix $\mathcal{H}$ is composed by the binary row vectors $\mathbf{v}_{m}$ for all $m=\left(\begin{array}{ll}a & b \\ c & d\end{array}\right) \in \mathcal{P}_{q}$. It is a matrix of $q^{3}+q$ rows and $q^{2}$ columns. If we view $m^{-1}$ as a map from $\mathbf{P}^{1}\left(\mathbf{F}_{q}\right)$ to $\mathbf{P}^{1}\left(\mathbf{F}_{q^{2}}\right)$ given by

$$
\left(\beta_{1}: \beta_{2}\right) \rightarrow\left(-d \beta_{1}+b \beta_{2}: c \beta_{1}-a \beta_{2}\right),
$$

then the $i$ th component of $v_{m}$ is 1 if and only if there is a point $P \in \mathbf{P}^{1}\left(\mathbf{F}_{q}\right)$ such that $m^{-1}(P)=\left(\alpha_{i}: 1\right)$. We define a binary vector $\mathbf{v}_{m}^{+}=\left(e_{1}, \ldots, e_{q^{2}}, e_{q^{2}+1}\right)$ for $m \in \mathcal{P}_{q}$, where $\left(e_{1}, \ldots, e_{q^{2}}\right)=\mathbf{v}_{m}$ and

$$
e_{q^{2}+1}= \begin{cases}1 & \text { if }(a: c) \in \mathbf{P}^{1}\left(\mathbf{F}_{q}\right) \\ 0 & \text { otherwise }\end{cases}
$$

One can verify that the last component of $\mathbf{v}_{m}^{+}$corresponds to whether there is a point $P \in$ $\mathbf{P}^{1}\left(\mathbf{F}_{q}\right)$ such that $m^{-1}(P)=(1: 0)=\infty$. The matrix $\mathcal{H}^{+}$is composed of the vectors $\mathbf{v}_{m}^{+}, m \in$ $\mathcal{P}_{q} . \mathcal{H}^{+}$is a matrix of $q^{3}+q$ rows and $q^{2}+1$ columns. All the row vectors have exactly $q+1$ many coordinates which are $1 \mathrm{~s}$.

Denote the lattices generated by the row vectors of $\mathcal{H}$ and $\mathcal{H}^{+}$by $\mathcal{L}(\mathcal{H})$ and $\mathcal{L}\left(\mathcal{H}^{+}\right)$ respectively. In $[\mathbf{3}]$, the authors showed that $\mathbf{v}_{1}=\left(q^{2}+q, \ldots, q^{2}+q\right) \in \mathcal{L}\left(\mathcal{H}^{+}\right)$and $\mathbf{v}_{2}=\left(q^{2}+q, q+1, \ldots, q+1\right) \in \mathcal{L}\left(\mathcal{H}^{+}\right)$.

Theorem 4. The vector $(1,0, \ldots, 0)$ is in the lattice $\mathcal{L}(\mathcal{H})$.

Note that most likely $\mathcal{L}(W)$ is a proper lattice of $\mathcal{L}(\mathcal{H})$, hence the above theorem does not imply that $(1,0, \ldots, 0)$ is in the lattice $\mathcal{L}(W)$ for any polynomial $W$.

Proof. Fix a $\gamma$ such that $\mathbf{F}_{q^{2}}=\mathbf{F}_{q}[\gamma]$. Firstly, observe that $\mathbf{v}_{3}=(1, \ldots, 1, q) \in \mathcal{L}\left(\mathcal{H}^{+}\right)$. This follows from $\mathbf{v}_{3}=\sum_{\beta \in \mathbf{F}_{q}} \mathbf{v}_{m_{\beta}} \in \mathcal{L}\left(\mathcal{H}^{+}\right)$, where $m_{\beta}=\left(\begin{array}{cc}1 & \beta \gamma \\ 0 & 1\end{array}\right) \in \mathcal{P}_{q}$. There are $q+1$ row vectors in $\mathcal{H}^{+}$such that both the first and the last coordinates are 1 . Since the projective linear map on a projective line is sharply 3 -transitive, a third coordinate with value 1 will uniquely determine the coset in $\mathcal{P}_{q}$. Thus the sum of these $q+1$ vectors is $\mathbf{v}_{4}=(q+1,1, \ldots, 1, q+1) \in \mathcal{L}\left(\mathcal{H}^{+}\right)$. 
From the above observations, we have

$$
\begin{gathered}
\mathbf{v}_{5}=\mathbf{v}_{2}-(q+1) \mathbf{v}_{3}=\left(q^{2}-1,0, \ldots, 0,1-q^{2}\right) \in \mathcal{L}\left(\mathcal{H}^{+}\right), \\
\mathbf{v}_{6}=\mathbf{v}_{4}-\mathbf{v}_{3}=(q, 0, \ldots, 0,1) \in \mathcal{L}\left(\mathcal{H}^{+}\right) .
\end{gathered}
$$

We deduce that

$$
\mathbf{v}_{7}=q \mathbf{v}_{6}-\mathbf{v}_{5}=\left(1,0, \ldots, 0, q^{2}+q-1\right) \in \mathcal{L}\left(\mathcal{H}^{+}\right),
$$

which implies $(1,0, \ldots, 0) \in \mathcal{L}(\mathcal{H})$.

\section{Concluding remarks and open problems}

In this paper, we study the validation of the heuristics made in the quasi-polynomial time algorithm solving the discrete logarithms in the small characteristic fields [3]. We find that the heuristics are problematic in the cases of non-Kummer extensions. We propose a few modifications to the algorithm, including some extra requirements for the polynomials $h_{0}$ and $h_{1}$, and a trap-avoiding descent strategy. The modified algorithm relies on three improved heuristics.

Proposition 1. If Heuristics 1, 2 and 3 hold, then the discrete logarithm problem over $\mathbf{F}_{q^{k}}$ $(k<q)$ can be solved in time $q^{O(\log (k))}$.

We believe that proving (or disproving) them are interesting open problems that help to understand the effectiveness of the new algorithm.

\section{References}

1. L. M. Adleman, 'A subexponential algorithm for the discrete logarithm problem with applications to cryptography', Proc. 20th IEEE Symp. on Foundations of Comp. Science (IEEE, 1979) 55-60.

2. L. M. Adleman, 'The function field sieve', Algorithmic number theory, Lecture Notes in Computer Science 877 (eds L. M. Adleman and M. D. A. Huang; Springer, 1994) 108-121.

3. R. Barbulescu, P. Gaudry, A. Joux and E. Thomé, 'A quasi-polynomial algorithm for discrete logarithm in finite fields of small characteristic', Cryptology ePrint Archive, Report 2013/400, 2013.

4. D. Coppersmith, 'Fast evaluation of logarithms in fields of characteristic two', IEEE Trans. Inform. Theory 30 (1984) no. 4, 587-594.

5. W. Diffie and M. E. Hellman, 'New directions in cryptography', IEEE Trans. Inform. Theory 6 (1976) 644-654.

6. T. ElGamal, 'A public key cryptosystem and a signature scheme based on discrete logarithms', IEEE Trans. Inform. Theory 33 (1985) 469-472.

7. A. Enge, 'A general framework for subexponential discrete logarithm algorithms in groups of unknown order', Finite geometries, Developments in Mathematics 3 (eds A. Blokhuis, J. W. P. Hirschfeld, D. Jungnickel and J. A. Thas; Kluwer, 2001) 133-146.

8. F. Göloglu, R. Granger, G. McGuire and J. Zumbrägel, 'On the function field sieve and the impact of higher splitting probabilities', Advances in cryptology - CRYPTO 2013, Lecture Notes in Computer Science 8043 (eds R. Canetti and J. A. Garay; Springer, 2013) 109-128.

9. D. M. Gordon, 'Discrete logarithms in GF(p) using the number field sieve', SIAM J. Discrete Math. 6 (1993) no. 1, 124-138.

10. M.-D. Huang and A. K. NArayanan, 'Finding primitive elements in finite fields of small characteristic', CoRR (2013), Preprint, 2013, arXiv:1304.1206 [cs.DM].

11. A. Joux, 'Faster index calculus for the medium prime case application to 1175-bit and 1425-bit finite fields', Advances in cryptology - EUROCRYPT 2013, Lecture Notes in Computer Science 7881 (eds T. Johansson and P. Q. Nguyen; Springer, 2013) 177-193.

12. A. Joux, 'A new index calculus algorithm with complexity $L(1 / 4+o(1))$ in very small characteristic', Cryptology ePrint Archive, Report 2013/095, 2013.

13. A. Joux and R. Lercier, 'The function field sieve in the medium prime case', Advances in cryptology EUROCRYPT 2006, Lecture Notes in Computer Science 4004 (ed. S. Vaudenay; Springer, 2006) 254-270. 
14. A. Joux, R. Lercier, N. Smart and F. Vercauteren, 'The number field sieve in the medium prime case', Advances in cryptology - CRYPTO 2006, Lecture Notes in Computer Science 4117 (Springer, 2006) 326-344.

15. R. Merkle, 'Secrecy, authentication, and public key systems', PhD Thesis, Stanford University, 1979.

16. D. Panario, X. Gourdon and P. Flajolet, 'An analytic approach to smooth polynominals over finite fields', Algorithmic number theory, Lecture Notes in Computer Science 1423 (ed. J. Buhler; Springer, 1998) 226-236.

17. J. Pollard, 'Monte Carlo methods for index computations ( $\bmod p)$ ', Math. Comp. 32 (1978) no. 143, 918-924.

18. D. WAN, 'Generators and irreducible polynomials over finite fields', Math. Comp. 66 (1997) no. 219, $1195-1212$.

\section{Qi Cheng}

School of Computer Science

University of Oklahoma

Norman, OK

USA

qcheng@cs.ou.edu

Jincheng Zhuang

School of Computer Science

University of Oklahoma

Norman, OK

USA

jzhuang@ou.edu

\author{
Daqing Wan \\ Department of Mathematics \\ University of California \\ Irvine, $C A$ \\ USA \\ dwan@math.uci.edu
}

